\title{
Costo de oportunidad en hombres que acuden a las unidades de medicina familiar en la ciudad de Querétaro, México
}

\author{
Edith Olimpia Martínez Carranza, ${ }^{1,2}$ Enrique Villarreal Ríos, ${ }^{1}$ \\ Emma Rosa Vargas Daza, ${ }^{1}$ Liliana Galicia Rodríguez ${ }^{1}$ \\ y Lidia Martínez González ${ }^{1}$
}

Forma de citar

Martínez Carranza EO, Villarreal Ríos E, Vargas Daza ER, Galicia Rodríguez L, Martínez González L. Costo de oportunidad en hombres que acuden a las unidades de medicina familiar en la ciudad de Querétaro, México. Rev Panam Salud Publica. 2010;28(6):456-62.

RESUMEN Objetivo. Determinar el costo de oportunidad en hombres que solicitan atención en las unidades de medicina familiar (UMF) del Instituto Mexicano del Seguro Social (IMSS) en la ciudad de Querétaro.

Métodos. Se seleccionó una muestra de 807 hombres de 20 a 59 años de edad que solicitaban atención en los servicios de medicina familiar, laboratorio y farmacia, proporcionados por UMF del IMSS en Querétaro. Se excluyeron los pacientes referidos a urgencias y los que se retiraron sin recibir atención. La muestra $(n=807)$ se calculó mediante la fórmula de promedios para población infinita, con un intervalo de confianza de 95\% (IC95\%) y un costo de oportunidad promedio de US\$ 5,5 para medicina familiar, US\$3,1 para laboratorio y de US\$ 2,3 para farmacia. Las estimaciones incluyeron el tiempo invertido en traslado, espera y atención; el número de acompañantes, y el costo del minuto para la actividad remunerada y no remunerada. El costo de oportunidad se calculó a través del costo por minuto estimado para traslado, espera y atención de pacientes y acompañantes.

Resultados. El costo de oportunidad correspondiente al traslado del paciente se estimó en US\$ 0,97 (IC95\%: 0,81-1,15), mientras que el de espera fue de US\$ 5,03 (IC95\%: 4,08-6,09) en medicina familiar, US\$ 0,06 (IC95\%: 0,05-0,08) en farmacia y US\$ 1,89 (IC95\%: 1,56$2,25)$ en laboratorio. El costo de oportunidad promedio cuando el paciente acudió sin compañía osciló entre US\$ 1,10 para el servicio de farmacia solo y US\$ 8,64 para medicina familiar, farmacia y laboratorio. El costo de oportunidad ponderado para medicina familiar fue de US\$ 6,24.

Conclusiones. Dado que el costo de oportunidad de los hombres que demandan servicios en las unidades de medicina familiar corresponde a más de la mitad de un salario mínimo, desde el enfoque institucional correspondería establecer si esa es la mejor alternativa de atención.

Palabras clave Análisis costo-beneficio; medicina familiar y comunitaria; salud del hombre; México.

Unidad de Investigación Epidemiológica y en Servicios de Salud Querétaro. Instituto Mexicano del Seguro Social. La correspondencia se debe dirigir a Enrique Villarreal Ríos, felibree@infosel.net.mx

2 Unidad de Medicina Familiar no. 16, Instituto Mexicano del Seguro Social, Querétaro, México.
En México, los cambios derivados de la propia dinámica demográfica, aunados a diversas acciones emprendidas por el sector salud, han contribuido a modificar en relativamente poco tiempo algunos aspectos fundamentales del perfil epidemiológico de la población (1).
Desde hace algunos años se ha incorporado la perspectiva económica como una herramienta poderosa para entender y actuar sobre la producción y el financiamiento de los servicios de salud $(2,3)$ El análisis económico ha pasado entonces a ocupar un lugar relevante a la hora 
de identificar problemas y plantear soluciones que ayuden a mejorar el desempeño de los sistemas de salud (4). Este nuevo enfoque investigativo revela que los costos de la atención médica presentan una situación muy peculiar que requiere de dos líneas de análisis: por un lado, los costos de producción de los servicios para el proveedor $\mathrm{y}$, por el otro, los costos de búsqueda y obtención del servicio para el consumidor (5).

Si se traslada el concepto de "costo de oportunidad" al campo de la salud, el valor monetario que se destina a la prevención o al tratamiento no es la cantidad de dinero gastado en la intervención, sino los beneficios que se dejan de obtener al no escoger la mejor alternativa. Por lo tanto, el camino a seguir es explorar todas las alternativas y elegir la que genere el máximo beneficio en función de costos para la sociedad $(6,7)$. En el caso de los trabajadores, esta pérdida de beneficios se puede evaluar a partir de sus ingresos salariales, considerando los tiempos de traslado y de espera en los servicios de salud como los principales indicadores para el análisis del costo de oportunidad (8-10).

Las necesidades de salud están determinadas por la cultura, el medio ambiente y la actividad desarrollada dentro de esos dos contextos, por lo que se trata de elementos que influyen directamente en los tipos de servicios de salud demandados por la población. Agregar por tanto la dimensión económica al análisis de las políticas y las medidas sanitarias brinda un conocimiento más integral del problema y contribuye incuestionablemente a una toma de decisiones más efectiva $(3,11)$.

En su etapa adulta, el hombre juega un papel importante en la población económicamente activa, que conlleva responsabilidades sociales, culturales y familiares (12). Datos de México revelan que en 2005 el grupo de hombres de 20 a 59 años de edad representaban $23,9 \%$ de la población total, pero daban cuenta de $51,7 \%$ de la población económicamente activa y de $80 \%$ de los hombres económicamente activos $(11,13)$.

Sobre ese mismo grupo etario se ha informado que, a lo largo de un año, 49,6\% acude por lo menos una vez a consulta externa de medicina familiar y 14,6\% a los servicios de laboratorio, pero no hay información disponible acerca del costo de oportunidad relacionado con estos servicios (14). El presente trabajo se pro- pone examinar precisamente el costo de oportunidad en hombres que solicitan atención en las unidades de medicina familiar (UMF) del Instituto Mexicano del Seguro Social (IMSS) en la ciudad de Querétaro.

\section{MATERIALES Y MÉTODOS}

Entre enero y octubre de 2009 se realizó un estudio de costo de oportunidad en una muestra de 807 hombres en edades de 20 a 59 años que solicitaban atención primaria en los servicios de medicina familiar, laboratorio y farmacia, proporcionados por las UMF no. 9, 13 y 16 del IMSS en la ciudad mexicana de Santiago de Querétaro.

La delegación IMSS en el estado de Querétaro atiende en 24 UMF a 917000 derechohabientes, más de $50 \%$ de la población total del estado, en tanto que el marco muestral de las tres unidades médicas estudiadas representa a $45 \%$ de la población de esta delegación y $60 \%$ de la población de su zona metropolitana adscrita al IMSS. El salario diario promedio de los trabajadores estudiados es de US $\$ 20,6,{ }^{3}$ cantidad superior al promedio nacional de US\$ 18,1 $(15,16)$. El estudio excluyó a los pacientes que se retiraron de la UMF sin recibir atención médica, así como a los referidos al servicio de urgencias de la UMF y al Hospital General Regional no. 1.

El tamaño de la muestra se estableció utilizando la fórmula de promedios para población infinita con un intervalo de confianza de 95\% (IC95\%) y un costo de oportunidad promedio de US\$ 5,5 para medicina familiar, US $\$ 3,1$ para laboratorio y US\$ 2,3 para farmacia, obteniéndose una muestra de 269 pacientes para cada una de las tres UMF participantes. A su vez, el total de la muestra se distribuyó de manera proporcional al número de usuarios de cada UMF, correspondiendo 78 pacientes por servicio a la UMF nueve, 102 pacientes por servicio a la 13 y 89 pacientes por servicio a la 16 .

En el servicio de medicina familiar se utilizó la técnica muestral por cuota, tomando como marco el listado de pacientes que acudieron a solicitar atención médica. Esa misma población se entrevistó en el servicio de farmacia y, en el

\footnotetext{
3 Calculado según el tipo de cambio de $\$ 12,7859$ por dólar estadounidense, establecido por el Banco de México (http://www.banxico.org.mx/), 2009.
}

caso del servicio de laboratorio, la muestra se obtuvo a partir del listado de pacientes con turnos para análisis.

Las variables sociodemográficas para los pacientes incluyeron edad, estado civil, ocupación y número (y ocupación) de acompañantes a la visita médica. El costo de oportunidad se contabilizó por minuto y se estimó considerando el tiempo absorbido - tanto para el paciente como para su acompañante- - por el traslado hasta la unidad médica, la espera en el lugar y la atención propiamente dicha.

\section{Tiempos de traslado, espera y atención}

\section{Tiempo de traslado}

El tiempo de traslado se calculó en base al promedio de minutos utilizados para viajar desde el lugar de partida del paciente hasta la unidad médica, multiplicado por dos para considerar el regreso.

\section{Tiempo de espera}

El tiempo de espera representó el promedio de minutos transcurridos entre el arribo del paciente a la unidad médica, al laboratorio o a la farmacia y el momento en que recibió la atención correspondiente.

\section{Tiempo de atención}

El tiempo de atención se calculó en base al promedio de minutos que pasaron desde el momento en que comenzó la atención hasta que finalizó - aplicado en cada uno de los servicios.

\section{Costo del minuto}

\section{Actividad laboral remunerada}

La actividad laboral remunerada se obtuvo preguntando directamente el salario del paciente ya sea por día, semana, quincena o mes, y convirtiéndolo luego al monto por minuto trabajado sobre la base de 8 horas diarias.

\section{Actividad laboral y estudiantil no remunerada}

Labores en la casa. El salario de las labores en el hogar se estipuló tomando como base el ingreso promedio de 8 horas diarias de la población con trabajos remunerados y contabilizándolo posteriormente por minuto. 
Labores estudiantiles. Para los menores de edad que acuden como acompañantes a las visitas médicas, la estimación de costos se realizó en dólares estadounidenses considerando la inversión familiar promedio anual (US\$ 57,32) para el sostenimiento por alumno reportada por la Secretaría de Educación Pública; dividida entre el número de días escolares (210 días), las horas que acuden a la escuela al día (5 horas) y posteriormente estimada por minuto (17).

\section{Estimación del costo de oportunidad}

El costo de oportunidad promedio vinculado a los tiempos invertidos por los pacientes -y sus acompañantesdurante el traslado, la espera y la atención, y también discriminados por servicios, se calculó del siguiente modo:

Traslado: tiempo estimado en el traslado multiplicado por el respectivo costo en minutos.

Costo de oportunidad promedio de la espera del paciente y sus acompañantes: tiempo de espera para recibir atención multiplicado por el respectivo costo en minutos.

Costo de oportunidad promedio de la atención del paciente y sus acompañantes: tiempo de atención multiplicado por el respectivo costo en minutos.

Costo de oportunidad promedio por servicio: suma del costo de oportunidad promedio en traslado, espera y atención por servicio.

Costo de oportunidad promedio ponderado: costo de oportunidad promedio por servicio ponderado, multiplicado por el porcentaje de población de pacientes con o sin acompañantes.

$$
\mathrm{CO} \mu=\sum_{i j=1}^{k} \mathrm{CO}_{i j}
$$

$\mathrm{CO} \mu=$ Costo de oportunidad promedio

$\sum_{i j=1}^{k} \mathrm{CO}_{i j}=\begin{aligned} & \text { costo de oportunidad del pa- } \\ & \text { ciente y acompañante uno, en }\end{aligned}$ los servicios de medicina familiar, laboratorio y farmacia.

$\mathrm{i}=$ Traslado, espera y atención

$\mathrm{j}=$ Paciente, acompañante 1

$1=$ Contabilizados desde el primero

$\mathrm{k}=$ Hasta el último

La " $\mathrm{i}$ " corresponde al traslado, espera y atención; la " $\mathrm{j}$ " corresponde al paciente y acompañante 1; contabilizados cada uno de ellos desde el primero "1" hasta el último " $\mathrm{k}$ ".

\section{RESULTADOS}

Los integrantes de la muestra del estudio tenían una media de edad de 40,2 años (IC95\%: 39,3-41,2), de los cuales 81,9\% (IC95\%: 77,3-86,5) vivían en pareja y 18,1\% (IC95: 13,6-22,8) vivían solos. La actividad predominante en los pacientes fue la de empleado $(71,9 \%$ [IC95\%: 66,5-77,3]) y, en los acompañantes, las labores del hogar (63,5\% [IC95\%: 50,4-76,6]) (cuadro 1). El 19,3\% (IC95\%: 14,6-24,0) acudió al servicio de salud con un acompañante y el resto lo hizo solo.

El tiempo total promedio invertido por paciente en el servicio de medicina familiar fue de 168,7 $\mathrm{min}$. (IC95\%: 153,2-184,2), en farmacia, 28,2 $\mathrm{min}$. (IC95\%: 26,1-30,4) y en laboratorio, 74,6 min. (IC95\%: 68,6-80,6). En el cuadro 2 se presentan los tiempos invertidos por pacientes y acompañantes, discriminados por servicio.

El costo de oportunidad promedio para el traslado del paciente fue de
US\$ 0,97 (IC95\%: 0,81-1,15), el de espera en la consulta de medicina familiar de US\$ 5,03 (IC95\%: 4,08-6,09) y el de atención, de US\$ 0,57 (IC95\%: 0,49-0,66). En farmacia el costo de oportunidad promedio totalizó US\$1,10 (IC95\%: 0,92-1,30) y en laboratorio, US\$ 2,91 (IC95\%: 2,41$3,46)$. En el cuadro 3 se presentan estos valores para pacientes y acompañantes, así como el costo de cada uno de los servicios analizados. En los casos de los pacientes que acudieron solos, el costo de oportunidad promedio osciló entre US\$1,10 y US\$ 8,64, mientras que el costo de oportunidad ponderado varió entre US\$ 1,07 para farmacia y un máximo de US\$ 8,19 cuando son utilizados conjuntamente los servicios de medicina familiar, farmacia y laboratorio (cuadro 4).

\section{DISCUSIÓN}

El análisis económico forma parte importante del proceso de producción de salud. En este sentido, el concepto de "costo de oportunidad" que se plantea en el presente trabajo permite examinar un área no explorada, al tomar en cuenta el costo que representa recibir atención para el usuario de los servicios de salud. $\mathrm{Si}$ bien en principio este concepto - costo de oportunidad- se refiere al costo de no haber elegido la mejor alternativa, posteriormente se ha vinculado también con el costo de la persona sustituta (persona que cubre y realiza las funciones del paciente en cuestión). En este sentido sería muy útil dar con una definición más completa para el concepto de costo de oportunidad, que contemple tanto el costo de la mejor oportunidad perdida como el costo del sustituto.

En el presente artículo se ha trabajado con ese concepto únicamente en relación a la mejor oportunidad perdida, un tema cuya discusión sin dudas aún sigue

CUADRO 1. Ocupación de la población estudiada que acude a las unidades de medicina familiar, Querétaro, México, 2009

\begin{tabular}{|c|c|c|c|c|c|c|}
\hline \multirow{3}{*}{$\frac{\text { Ocupación }}{\text { Actividades en casa }}$} & \multicolumn{3}{|c|}{ Paciente } & \multicolumn{3}{|c|}{ Acompañante } \\
\hline & \multirow{2}{*}{$\frac{\text { Porcentaje }}{0,4}$} & \multicolumn{2}{|c|}{ IC95\% $\%^{a}$} & \multirow{2}{*}{$\frac{\text { Porcentaje }}{63,5}$} & \multicolumn{2}{|c|}{ IC95\% } \\
\hline & & 0,0 & 1,2 & & 50,4 & 76,6 \\
\hline Empleado(a) & 71,9 & 66,5 & 77,3 & 26,9 & 14,8 & 39,0 \\
\hline Obrero(a) & 13,0 & 9,0 & 17,0 & 3,8 & 0,0 & 9,0 \\
\hline Profesional & 7,4 & 4,3 & 10,5 & 3,8 & 0,0 & 9,0 \\
\hline Estudiante & 0,0 & 0,0 & 0,0 & 1,9 & 0,0 & 5,6 \\
\hline Comerciante & 4,4 & 2,0 & 6,8 & 0,0 & 0,0 & 0,0 \\
\hline Pensionado & 3,0 & 1,0 & 3,0 & 0,0 & 0,0 & 0,0 \\
\hline
\end{tabular}


CUADRO 2. Tiempo de traslado, espera y atención del paciente y acompañante que acuden a las unidades de medicina familiar, por servicio utilizado, Querétaro, México, 2009a

\begin{tabular}{|c|c|c|c|c|c|c|c|c|c|c|c|c|}
\hline \multirow{2}{*}{$\frac{\text { Usuario }}{\text { Medicina familiar }}$} & \multicolumn{3}{|c|}{ Traslado } & \multicolumn{3}{|c|}{ Espera } & \multicolumn{3}{|c|}{ Atención } & \multicolumn{3}{|c|}{ Total } \\
\hline & Media & \multicolumn{2}{|c|}{ IC95\% ${ }^{b}$} & Media & \multicolumn{2}{|c|}{ IC95\% } & Media & \multicolumn{2}{|c|}{ IC95\% } & Media & \multicolumn{2}{|c|}{ IC95\% } \\
\hline $\begin{array}{l}\text { Paciente } \\
\text { Acompañante (uno) }\end{array}$ & $\begin{array}{l}24,9 \\
30,8\end{array}$ & $\begin{array}{l}23,1 \\
24,8\end{array}$ & $\begin{array}{l}26,7 \\
36,8\end{array}$ & 129,0 & 116,1 & 142,0 & 14,7 & 14,0 & 15,5 & $\begin{array}{l}168,7 \\
174,6\end{array}$ & $\begin{array}{l}153,2 \\
154,8\end{array}$ & $\begin{array}{l}184,2 \\
194,3\end{array}$ \\
\hline $\begin{array}{l}\text { Farmacia } \\
\text { Paciente } \\
\text { Acompañante (uno) }\end{array}$ & $\begin{array}{l}24,9 \\
30,8\end{array}$ & $\begin{array}{l}23,1 \\
24,8\end{array}$ & $\begin{array}{l}26,7 \\
36,8\end{array}$ & 1,6 & 1,4 & 1,8 & 1,7 & 1,5 & 1,9 & $\begin{array}{l}28,2 \\
34,1\end{array}$ & $\begin{array}{l}26,1 \\
27,7\end{array}$ & $\begin{array}{l}30,4 \\
40,5\end{array}$ \\
\hline $\begin{array}{l}\text { Laboratorio } \\
\text { Paciente } \\
\text { Acompañante (uno) }\end{array}$ & $\begin{array}{l}24,9 \\
30,8\end{array}$ & $\begin{array}{l}23,1 \\
24,8\end{array}$ & $\begin{array}{l}26,7 \\
36,8\end{array}$ & 48,4 & 44,3 & 52,6 & 1,2 & 1,2 & 1,3 & $\begin{array}{l}74,6 \\
80,5\end{array}$ & $\begin{array}{l}68,6 \\
70,2\end{array}$ & $\begin{array}{l}80,6 \\
90,7\end{array}$ \\
\hline
\end{tabular}

Fuente: elaboración de los autores.

a La cantidad de tiempo está expresada en minutos.

b IC95\%: intervalo de confianza de 95\%.

CUADRO 3. Costo de oportunidad de traslado, espera y atención del paciente y acompañante por tipo de servicio utilizado en las unidades de medicina familiar, Querétaro, México, 2009

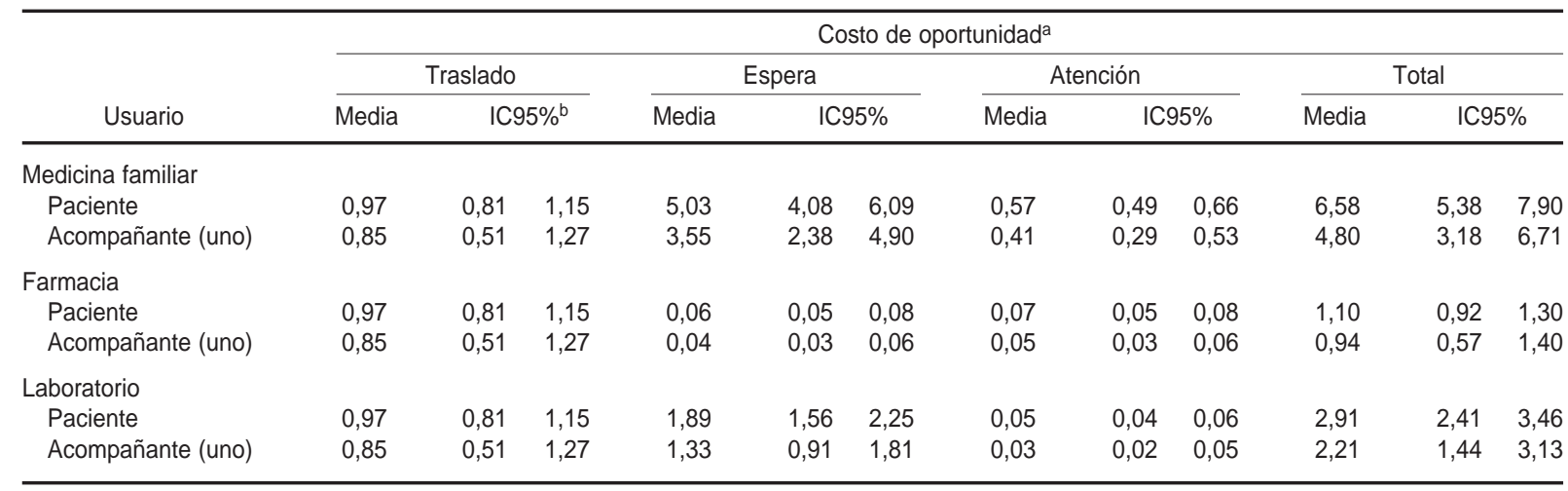

Fuente: elaboración de los autores.

a Costos expresados en dólares estadounidenses, de acuerdo al tipo de cambio establecido por el Banco de México [http://www.banxico.org.mx/ (US\$1 = \$12,78)], 2009.

b IC95\%: intervalo de confianza de $95 \%$.

CUADRO 4. Costo de oportunidad promedio y ponderado por escenario del paciente y acompañante que acuden a las unidades de medicina familiar, Querétaro, México, 2009

\begin{tabular}{|c|c|c|c|}
\hline \multirow[b]{2}{*}{ Servicio } & \multicolumn{2}{|c|}{$\begin{array}{c}\text { Costo de oportunidad } \\
\text { promedio }^{a}\end{array}$} & \multirow{2}{*}{$\begin{array}{c}\text { Costo de } \\
\text { oportunidad } \\
\text { ponderado }^{a}\end{array}$} \\
\hline & Paciente & Acompañante & \\
\hline Medicina familiar & 6,58 & 4,80 & 6,24 \\
\hline Farmacia & 1,10 & 0,94 & 1,07 \\
\hline Laboratorio & 2,91 & 2,21 & 2,77 \\
\hline Medicina familiar y Farmacia & 6,71 & 4,90 & 6,36 \\
\hline Medicina familiar y Laboratorio & 8,52 & 6,17 & 8,06 \\
\hline Farmacia y Laboratorio & 3,04 & 2,31 & 2,90 \\
\hline Medicina familiar, Farmacia y Laboratorio & 8,64 & 6,26 & 8,19 \\
\hline
\end{tabular}

abierta ¿Cuál es entonces el costo de la mejor oportunidad perdida? En este trabajo se propone como el costo que implica acudir a la unidad médica, es decir, el costo que tiene lo que se deja de reali- zar en la actividad habitual del paciente y/o acompañante. $\mathrm{Al}$ respecto se puede señalar que cuando se expide una incapacidad por enfermedad general el trabajador recibe $60 \%$ de su salario, lo que podría llevar a pensar que la estimación del costo de oportunidad debería realizarse sobre el $40 \%$ del salario no percibido. No obstante, también sucede que cuando se dictamina una incapacidad 
por riesgo de trabajo el porcentaje que el trabajador percibe es del 100\% (del salario). Si se utiliza esa misma lógica, el costo de oportunidad debería estimarse en cero pesos, puesto que el porcentaje que se deja de percibir en este caso es del $0 \%$. Como tal situación se aleja del concepto económico del costo de oportunidad, se decidió estimar este costo en base al salario nominal.

Para la asignación del salario a las personas con actividad laboral no remunerada, específicamente las actividades en el hogar, se empleó el salario promedio notificado para la población que dentro de este estudio percibe remuneración económica. Aquí cabe mencionar que la población sin actividad económica remunerada correspondió a la pareja del paciente y se asumió que comparte las mismas características socioculturales que la que percibe un salario y por lo tanto estaría en condiciones de recibir un salario similar en caso de ejercer una actividad laboral remunerada.

En cuanto a los escolares que acuden como acompañantes del paciente, la asignación más objetiva podría ser el monto que la familia invierte en cada escolar, de ahí que se haya empleado el monto notificado por la Secretaría de Educación Pública, aunque es obvio que no se trata de la única posibilidad. La proporción de acompañantes para la población de este estudio fue relativamente baja, lo cual podría deberse al hecho de que es una población joven, económicamente activa y sin problemas físicos. En el caso de la población femenina, este comportamiento fue diferente ya sea por tener bajo custodia a un menor o por condiciones físicas que limitaron su autonomía (18). Como parte de la metodología, se contabilizó el tiempo de traslado del usuario y acompañante(s) desde su lugar de salida a la unidad médica y viceversa, pues de otro modo se tendría una evaluación parcial que impactaría directamente en el costo de oportunidad. Este indicador ya se ha utilizado para evaluar el costo de oportunidad de la atención prenatal (18).

Los tiempos invertidos en la espera para recibir atención en el servicio de medicina familiar fueron distintos a los establecidos institucionalmente, en particular los correspondientes a la cita previa (19). Tal diferencia pareciera responder a que en el presente estudio los pacientes -y sus acompañantes- fueron quienes decidieron cuándo arribar a la unidad mé- dica, o bien se presentaron espontáneamente, dilatando por razones obvias el tiempo de espera (20). De todas maneras, lo importante es que los tiempos de espera notificados en este trabajo corresponden a los minutos realmente invertidos por el paciente para recibir atención.

El costo de oportunidad en cada uno de los servicios presentó variaciones entre el paciente y el acompañante, hecho debido al tipo de actividad que desarrolla la población, aun cuando a la población sin actividad remunerada se le asignó el promedio de la población que sí percibe ingresos por la actividad que desempeña. El costo de oportunidad por la espera para la consulta de medicina familiar fue mayor que para el laboratorio. En farmacia, el costo de oportunidad se redujo considerablemente por la capacidad instalada y el tipo de acciones realizadas en el servicio.

La comparación del costo de oportunidad del tiempo de atención entre los servicios analizados mostró gran variación por las características propias de la organización de cada uno de ellos. Un ejemplo fue el servicio de farmacia, que registró una respuesta rápida por el tipo de producto solicitado. Algo similar ocurrió con el laboratorio donde el personal de salud trabajó con el paciente en la toma de muestra y, posteriormente, en el análisis de la muestra recolectada, ya no fue necesaria la presencia del paciente. En medicina familiar se presentó una situación muy diferente, puesto que la participación del paciente fue indispensable para establecer el diagnóstico y el tratamiento.

En este trabajo el costo de oportunidad se ha calculado sobre la base del paciente que genera una consulta o un servicio, por lo cual se puede hablar de costo de oportunidad por consulta. No obstante, si la estimación se realizara para la atención de una fractura o alguna otra patología que demandara varias visitas al servicio, el costo de la atención sería superior. El costo de oportunidad promedio y ponderado de los escenarios analizados es una herramienta que puede permitir planificar los servicios de salud teniendo en cuenta la perspectiva del paciente, y no sólo la perspectiva institucional, en pos de una utilización más racional de los recursos existentes.

Dentro de las tres áreas identificadas en relación al costo de oportunidad del paciente - el traslado, la espera y la atención-, es poco lo que puede hacerse en cuanto al costo del traslado dado que las unidades médicas están localizadas estratégicamente a una distancia determinada del domicilio de cada paciente. Una opción podría ser mejorar la eficiencia de los sistemas de transporte público, pero tal medida daría una solución relativa y seguramente no sería costo-efectiva.

Un escenario diferente se presenta para el costo de oportunidad de la espera y la atención. En cuanto a la espera, se podrían diseñar nuevas funciones de producción que permitieran tener procesos más eficientes y, en consecuencia, reducir el costo de oportunidad. Una posibilidad sería la atención "en banda", es decir que el paciente sea atendido un mismo día en todos los servicios requeridos, optimizando así los tiempos de espera. Indirectamente, al evitar viajes innecesarios, la atención en banda también reduciría el costo de oportunidad de transporte. Por otro lado, la conveniencia de reducir el costo de oportunidad de la atención es cuanto menos cuestionable. De hecho, tal reducción implicaría una menor estancia del paciente con el médico, es decir una atención más corta, lo cual en principio y desde un punto de vista clínico no pareciera ser aconsejable.

El costo de oportunidad total de los hombres que demandan atención corresponde aproximadamente a más de un salario mínimo diario de US\$ 4,3 para el área geográfica " $\mathrm{C}$ ", semejante al del grupo de mujeres $(21,22)$. Para efecto de la aplicación de salarios mínimos, México se ha dividido en tres áreas geográficas - "A", "B" y " $C$ ". Cada una tiene un salario mínimo general y 73 salarios mínimos profesionales. Asimismo, el país está organizado en 2456 divisiones políticas: 2440 municipios y 16 delegaciones. El 2,65\% de dichas divisiones políticas se ubican en el área " $\mathrm{A}$ ", 2,24\% en la "B" y 95,11\% en la "C" (23).

La metodología aquí utilizada permitió conocer el costo de oportunidad ponderándolo a partir de tres variables: el costo del minuto, el tiempo invertido y el porcentaje de pacientes que acuden acompañados, resultando en un valor más cercano a la realidad, dado que generalmente solo se contabiliza la inversión del paciente.

Aun cuando aquí el costo de oportunidad se estimó para una sola visita, los valores resultantes podrían servir de base para calcular el costo para la atención de un padecimiento que requiere dos, tres o más visitas al centro de salud, 
como podría ser el caso de los pacientes con diagnóstico de fractura, diabetes, hipertensión o embarazo.

Una limitación que tuvo el estudio radica en que el costo de oportunidad estimado no es representativo de todo el país, por lo que si se quisiera extrapolar a otras poblaciones debería ser ajustado. Esto es así porque si bien los tiempos de traslado, espera y atención, así como el porcentaje de acompañantes, deberían ser similares a los de otras partes puesto que se trata de la misma institución de salud, no sucede lo mismo con los perfiles económicos de las poblaciones que varían entre las áreas geográficas del país. Queda claro entonces que si el muestreo hubiera incluido conglomerados representativos de diferentes zonas geográficas, los resultados alcanzarían otras dimensiones.

La situación actual de la atención de salud ofrece una valiosa posibilidad de explorar nuevas opciones que podrían generar aún mayores beneficios para la sociedad. Dado que el costo de oportuni- dad de los hombres que demandan servicios en las unidades de medicina familiar corresponde a más de la mitad de un salario mínimo, desde el enfoque institucional correspondería establecer si esa es la mejor alternativa de atención. En este sentido, los directivos institucionales deben tomar en cuenta que el gasto en atención médica involucra no solo lo invertido por la institución, sino además el costo de oportunidad del usuario de dichos servicios.

\section{REFERENCIAS}

1. Fundación Mexicana para la salud. La salud en México: 2006/2012. Visión de FUNSALUD. Hallado en: http:/ / www.funsalud. org.mx/vision_2006-2012/documentos / VISION\%20SALUD.pdf. Acceso el 13 de diciembre de 2010.

2. Navarro RF, Hernández SA, Pérez RA, Cicero SR. Costo-efectividad de los métodos diagnósticos de la tuberculosis. Rev Inst Nal Enf Resp Mex. 2006;19(3):214-21.

3. González PE, Gutiérrez DC, Stevens G, Barraza Ll, Porras CR, Carvalho N, et al. Definición de prioridades para las intervenciones de salud en el sistema de protección social en salud de México. Salud Publica Mex. 2007;49(1):38-52.

4. Collazo HM, Cárdenas RJ, González LR, Miyar AR, Gálvez GA, Cosme CJ. La economía de la salud: ¿debe ser de interés para el campo sanitario? Rev Panam Salud Publica. 2002;12(5):359-65.

5. Arredondo A, Nájera P, Leyva R. Atención médica ambulatoria en México: el costo para los usuarios. Salud Publica Mex. 1999;41(1): 18-26.

6. Valencia JE. Los indicadores para los estudios de costos: Costo de oportunidad por consumo de sustancias psicoactivas nivel 2: Indicadores de costos indirectos. Hallado en: http: / / www.cicad.oas.org/OID/new / research/Costs /N2\% 208\%20Costo $\% 20$ de $\% 200$ portunidad\%20JEV.pdf. Acceso el 8 de octubre de 2010.

7. Barbieri NC. Estado y mercado en salud. Una aproximación desde la economía de la salud. Hallado en: http://medicina.uncoma.edu. ar/download/postgrado/gestion_auditoria/ bibliografia/modulo_04/estado_y_mer cado_en_salud.pdf. Acceso el 13 de diciembre de 2010.

8. Rodríguez-Ledesma MA, Vidal-Rodríguez C. Conceptos básicos de economía de la salud para el médico general. Rev Med Inst Mex Seguro Soc. 2007;45(5):523-32.
9. Collazo HM, Cárdenas RJ, Cosme CJ, González LR, Rolando MA. La economía de la salud: ¿debe ser de interés para el campo sanitario?. Hallado en: http://www.scielo sp.org/pdf/rpsp/v12n5/14098.pdf. Acceso el 6 de diciembre del 2010.

10. Baly GA, Toledo RM, Rodríguez JF. La economía de la salud, la eficiencia y el costo de oportunidad. Rev Cubana Med Gen Integr. 2001;17(4):395-8.

11. Puig JJ, Pinto PJL. El coste de oportunidad del tiempo remunerado en la producción de salud [documento de trabajo]. Hallado en: http://www.econ.upf.edu/ puig/publica cions/paper39.pdf. Acceso el 8 de octubre de 2010.

12. Fernández SN. El adulto. Hallado en: http:// www.tuobra.unam.mx/publicadas / 021123215354-.html. Acceso el 8 de octubre de 2010.

13. Instituto Nacional de Estadística y Geografía, México. II Conteo nacional de población y vivienda 2005. Pirámide de Población 2005. México. Hallado en: http://www.inegi.org. $\mathrm{mx} /$ est/contenidos/espanol/sistemas/cgpv 2000/100historia $/$ epobla28.asp?s=est\&c=971 9. Acceso el 16 de febrero del 2010.

14. Torres LG, Villarreal RE, Martínez GL, Vargas DER, Galicia RL, Hernández CJL, et al. Perfil de uso de los servicios de salud en primer nivel de atención por hombres adultos jóvenes (de 20 a 59 años). En: Villarreal RE, Martínez GL, Galicia RL, Vargas DER, eds. El sistema de salud, perfil de uso y costo en primer nivel de atención. México: Universidad Autónoma de Querétaro, Instituto Mexicano del Seguro Social; 2007. Pp. 35-42.

15. Comisión Nacional de los Salarios Mínimos, México. Salario promedio diario de cotización por entidad federativa según mes, enero-junio de 2010. Hallado en: http:// www.conasami.gob.mx/pdf/entidad $\% 20$ federativa/SALPROFENTFED_2010.pdf. Acceso el 8 de octubre de 2010.
16. Instituto Mexicano del Seguro Social. Información de la delegación. Hallado en: http://www.imss.gob.mx/Delegaciones/ Queretaro/imss_en_queretaro.htm. Acceso el 8 de octubre de 2010.

17. Secretaría de Educación Pública, México. Reportes interactivos. Estadística en la Educación Básica. Hallado en: http://sieeb. basica.sep.gob.mx/\#. Acceso el 19 de octubre del 2008.

18. Sanabria RG, Gálvez GA, Álvarez MM. Costos para la mujer durante la atención prenatal. Rev Cubana Salud Publica. 2004;30(1): 77-82.

19. Instituto Mexicano del Seguro Social. Seguridad y Solidaridad Social. Programa cita previa. Hallado en: http://www.imss.gob.mx/ Delegaciones/Coahuila/IMSSenCoahuila/ cita_previa_coah.htm. Acceso el 6 diciembre del 2010.

20. Maldonado IG, Fragoso BJ, Orrisco TS, Flores VM, Quiróz PC, Sandoval PA. Tiempo de espera en el primer nivel para la población asegurada por el IMSS. Rev Med IMSS. 2002;40 (5):421-9.

21. Comisión Nacional de los Salarios Mínimos, México. Salarios mínimos vigentes a partir del 1 de enero de 2010. México: Diario Oficial de la Federación (23 de diciembre); 2009.

22. García AL. Costo oportunidad en mujeres en primer nivel de atención. Síntesis ejecutiva. México: Unidad de Investigación Epidemiológica y en Servicios de Salud Querétaro; 2008.

23. Comisión Nacional de Salarios Mínimos, México. División de la República Mexicana en áreas geográficas. Hallado en: http:// www.conasami.gob.mx/div_sal_min.html. Acceso el 6 diciembre de 2010.

Manuscrito recibido el 16 de febrero de 2010. Aceptado para publicación, tras revisión, el 6 de octubre de 2010. 
ABSTRACT Objective. To determine the opportunity cost for men who seek care in the family medicine units (FMU) of the Mexican Social Security Institute (IMSS, Instituto Mexicano del Seguro Social) in the city of Querétaro.

\section{Opportunity cost for men who visit family medicine units in the city of Querétaro, Mexico}

Methods. A sample was selected of 807 men, ages 20 to 59 years, who sought care through the family medicine, laboratory, and pharmacy services provided by the FMU at the IMSS in Querétaro. Patients referred for emergency services and those who left the facilities without receiving care were excluded. The sample $(n=807)$ was calculated using the averages for an infinite population formula, with a confidence interval of $95 \%$ (CI95\%) and an average opportunity cost of US\$5.5 for family medicine, US $\$ 3.1$ for laboratory services, and US $\$ 2.3$ for pharmacy services. Estimates included the amount of time spent on travel, waiting, and receiving care; the number of people accompanying the patient, and the cost per minute of paid and unpaid job activities. The opportunity cost was calculated using the estimated cost per minute for travel, waiting, and receiving care for patients and their companions.

Results. The opportunity cost for the patient travel was estimated at US $\$ 0.97$ (CI95\%: 0.81-1.15), while wait time was US\$5.03 (CI95\%: 4.08-6.09) for family medicine, US\$0.06 (CI95\%: 0.05-0.08) for pharmacy services, and US\$1.89 (CI95\%: 1.56-2.25) for laboratory services. The average opportunity cost for an unaccompanied patient visit varied between US $\$ 1.10$ for pharmacy services alone and US\$8.64 for family medicine, pharmacy, and laboratory services. The weighted opportunity cost for family medicine was US\$6.24.

Conclusions. Given that the opportunity cost for men who seek services in FMU corresponds to more than half of a minimum salary, it should be examined from an institutional perspective whether this is the best alternative for care.

Key words Cost-benefit analysis; family practice; men's health; Mexico. 